\title{
Identities involving harmonic and hyperharmonic numbers
}

\author{
Dae San Kim ${ }^{1}$ and Taekyun Kim²*
}

"Correspondence: tkkim@kw.ac.kr ${ }^{2}$ Department of Mathematics, Kwangwoon University, Seoul, 139-701, Republic of Korea Full list of author information is available at the end of the article

\begin{abstract}
In this paper, we give some new and interesting identities involving harmonic and hyperharmonic numbers which are derived from the transfer formula for the associated sequences.
\end{abstract}

\section{Introduction}

Let $\mathcal{F}$ be the set of all formal power series in the variable $t$ over $\mathbf{C}$ with

$$
\mathcal{F}=\left\{f(t)=\sum_{k=0}^{\infty} \frac{a_{k}}{k !} t^{k} \mid a_{k} \in \mathbf{C}\right\} .
$$

Suppose that $\mathbb{P}$ is the algebra of polynomials in the variable $x$ over $\mathbf{C}$ and that $\mathbb{P}^{*}$ is the vector space of all linear functionals on $\mathbb{P}$. The action of the linear functional $L$ on a polynomial $p(x)$ is denoted by $\langle L \mid p(x)\rangle$.

Let $f(t) \in \mathcal{F}$. Then we consider a linear functional on $\mathbb{P}$ by setting

$$
\left\langle f(t) \mid x^{n}\right\rangle=a_{n} \quad(n \geq 0)(\text { see }[1,2]) .
$$

From (1) and (2), we note that

$$
\left\langle t^{k} \mid x^{n}\right\rangle=n ! \delta_{n, k} \quad(n, k \geq 0)(\text { see }[1,3-5])
$$

where $\delta_{n, k}$ is the Kronecker symbol.

Let $f_{L}(t)=\sum_{k=0}^{\infty} \frac{\left\langle L \mid x^{n}\right\rangle}{k !} t^{k}$. Then we see that $\left\langle f_{L}(t) \mid x^{n}\right\rangle=\left\langle L \mid x^{n}\right\rangle$. The map $L \longmapsto f_{L}(t)$ is a vector space isomorphism from $\mathbb{P}^{*}$ onto $\mathcal{F}$. Henceforth, $\mathcal{F}$ is thought of as both a formal power series and a linear functional. We call $\mathcal{F}$ the umbral algebra. The umbral calculus is the study of umbral algebra. The order $O(f(t))$ of the nonzero power series $f(t)$ is the smallest integer $k$ for which the coefficient of $t^{k}$ does not vanish. If $O(f(t))=0$, then $f(t)$ is called an invertible series. If $O(f(t))=1$, then $f(t)$ is called a delta series. Let $O(f(t))=1$ and $O(g(t))=0$. Then there exists a unique sequence $s_{n}(x)$ of polynomials such that $\left\langle g(t) f(t)^{k} \mid s_{n}(x)\right\rangle=n ! \delta_{n, k}$ for $n, k \geq 0$. The sequence $s_{n}(x)$ is called the Sheffer sequence for $(g(t), f(t))$ which is denoted by $s_{n}(x) \sim(g(t), f(t))$ (see $\left.[1,3,6]\right)$. If $s_{n}(x) \sim(1, f(t))$, then $s_{n}(x)$ is called the associated sequence for $f(t)$. By (3), we easily see that $\left\langle e^{y t} \mid p(x)\right\rangle=p(y)$.

C) $2013 \mathrm{Kim}$ and Kim; licensee Springer. This is an Open Access article distributed under the terms of the Creative Commons Attribution License (http://creativecommons.org/licenses/by/2.0), which permits unrestricted use, distribution, and reproduction in any medium, provided the original work is properly cited. 
Let $f(t) \in \mathcal{F}$ and $p(x) \in \mathbb{P}$. Then we have

$$
f(t)=\sum_{k=0}^{\infty} \frac{\left\langle f(t) \mid x^{k}\right\rangle}{k !} t^{k}, \quad p(x)=\sum_{k=0}^{\infty} \frac{\left\langle t^{k} \mid p(x)\right\rangle}{k !} x^{k} \quad(\text { see }[1,6,7]) .
$$

From (4), we note that

$$
p^{(k)}(0)=\left\langle t^{k} \mid p(x)\right\rangle, \quad\left\langle 1 \mid p^{(k)}(x)\right\rangle=p^{(k)}(0) .
$$

By (5), we easily see that

$$
t^{k} p(x)=p^{(k)}(x)=\frac{d^{k} p(x)}{d x^{k}} \quad(k \geq 0)(\text { see }[2,3,6,7])
$$

Let $\phi_{n}(x)$ be exponential polynomials which are given by

$$
\sum_{k=0}^{\infty} \frac{\phi_{k}(x)}{k !} t^{k}=e^{x\left(e^{t}-1\right)} \quad(\text { see }[2,6,8])
$$

Thus, by (7), we get

$$
\phi_{n}(x)=\sum_{k=0}^{n} S_{2}(n, k) x^{k} \sim(1, \log (1+t))
$$

where $S_{2}(n, k)$ is the Stirling number of the second kind.

The Stirling number of the first kind is defined by

$$
(x)_{n}=x(x-1) \cdots(x-n+1)=\sum_{k=0}^{n} S_{1}(n, k) x^{k} .
$$

Thus, by (9), we get

$$
S_{1}(n, k)=\frac{1}{k !}\left\langle t^{k} \mid(x)_{n}\right\rangle \quad(\text { see }[2,5])
$$

Let $p_{n}(x) \sim(1, f(t)), q_{n}(x) \sim(1, g(t))$. Then the transfer formula for the associated sequences is given by

$$
q_{n}(x)=x\left(\frac{f(t)}{g(t)}\right)^{n} x^{-1} p_{n}(x) \quad(\text { see }[2,8]) .
$$

The $n$th harmonic number is $H_{n}=\sum_{i=1}^{n} \frac{1}{i}(n \geq 1)$ and $H_{0}=0$.

In general, the hyperharmonic number $H_{n}^{(r)}$ of order $r$ is defined by

$$
H_{n}^{(r)}=\left\{\begin{array}{ll}
0 & \text { if } n \leq 0 \text { or } r<0, \\
\frac{1}{n} & \text { if } r=0, n \geq 1, \\
\sum_{i=1}^{n} H_{i}^{(r-1)} & \text { if } r, n \geq 1
\end{array} \quad \text { (see }[9,10]\right)
$$


From (12), we note that $H_{n}^{(1)}$ is the ordinary harmonic number $H_{n}$. It is known that

$$
H_{n}^{(r)}=\left(\begin{array}{c}
n+r-1 \\
r-1
\end{array}\right)\left(H_{n+r-1}-H_{r-1}\right) \quad(\text { see }[9,10]) .
$$

The generating functions of the harmonic and hyperharmonic numbers are given by

$$
\sum_{n=1}^{\infty} H_{n} t^{n}=-\frac{\log (1-t)}{1-t}
$$

and

$$
\sum_{n=1}^{\infty} H_{n}^{(r)} t^{n}=-\frac{\log (1-t)}{(1-t)^{r}}, \quad \text { respectively. }
$$

The purpose of this paper is to give some new and interesting identities involving harmonic and hyperharmonic numbers which are derived from the transfer formula for the associated sequences.

\section{Identities involving harmonic and hyperharmonic numbers}

From (7) and (8), we note that

$$
\phi_{n}(x)=\sum_{j=0}^{n} S_{2}(n, j) x^{j} \sim(1, \log (1+t))
$$

and

$$
(-1)^{n} \phi_{n}(-x) \sim(1,-\log (1-t)) .
$$

Let us assume that

$$
q_{n}(x) \sim\left(1, t(1-t)^{r}\right) .
$$

From (11), (18) and $x^{n} \sim(1, t)$, we note that

$$
\begin{aligned}
q_{n}(x) & =x\left(\frac{t}{t(1-t)^{r}}\right)^{n} x^{-1} x^{n}=x(1-t)^{-r n} x^{n-1} \\
& =x \sum_{k=0}^{n-1}\left(\begin{array}{c}
-r n \\
k
\end{array}\right)(-t)^{k} x^{n-1}=x \sum_{k=0}^{n-1}\left(\begin{array}{c}
r n+k-1 \\
k
\end{array}\right) t^{k} x^{n-1} \\
& =x \sum_{k=0}^{n-1}\left(\begin{array}{c}
r n+k-1 \\
k
\end{array}\right)(n-1)_{k} x^{n-1-k}=\sum_{k=1}^{n-1}\left(\begin{array}{c}
r n+k-1 \\
k
\end{array}\right)(n-1)_{k} x^{n-k} \\
& =\sum_{k=1}^{n}\left(\begin{array}{c}
r n+n-k-1 \\
n-k
\end{array}\right)(n-1)_{n-k} x^{k} .
\end{aligned}
$$

Now, we use the following fact:

$$
\sum_{n=1}^{\infty} H_{n}^{(r)} t^{n}=-\frac{\log (1-t)}{(1-t) r}
$$


For $n \geq 1$, by (11), (17) and (18), we get

$$
\begin{aligned}
q_{n}(x) & =x\left(\frac{-\log (1-t)}{t(1-t)^{r}}\right)^{n} x^{-1}(-1)^{n} \phi_{n}(-x) \\
& =x\left(\sum_{l=0}^{\infty} H_{l+1}^{(r)} t^{l}\right)^{n} x^{-1}(-1)^{n} \sum_{j=1}^{n} S_{2}(n, j)(-x)^{j} \\
& =(-1)^{n} \sum_{j=1}^{n} S_{2}(n, j)(-1)^{j} x\left(\sum_{l=0}^{\infty} H_{l+1}^{(r)} t^{l}\right)^{n} x^{j-1} \\
& =(-1)^{n} \sum_{j=1}^{n} S_{2}(n, j)(-1)^{j} x\left(\sum_{l=0}^{j-1}\left(\sum_{l_{1}+\cdots+l_{n}=l} H_{l_{1}+1}^{(r)} \cdots H_{l_{n}+1}^{(r)}\right) t^{l}\right) x^{j-1} \\
& =(-1)^{n} \sum_{j=1}^{n} \sum_{l=0}^{j-1} \sum_{l_{1}+\cdots+l_{n}=l} S_{2}(n, j)(-1)^{j} H_{l_{1}+1}^{(r)} \cdots H_{l_{n}+1}^{(r)}(j-1)_{l} x^{j-l} \\
& =(-1)^{n} \sum_{j=1}^{n} \sum_{k=1}^{j} \sum_{l_{1}+\cdots+l_{n}=j-k} S_{2}(n, j)(-1)^{j} H_{l_{1}+1}^{(r)} \cdots H_{l_{n}+1}^{(r)}(j-1)_{j-k} x^{k} \\
& \left.=(-1)^{n} \sum_{k=1}^{n} \sum_{j=k}^{n} \sum_{l_{1}+\cdots+l_{n}=j-k}(-1)^{j} S_{2}(n, j) H_{l_{1}+1}^{(r)} \cdots H_{l_{n}+1}^{(r)}(j-1)_{j-k}\right\} x^{k} .
\end{aligned}
$$

Therefore, by comparing coefficients on both sides of (19) and (20), we obtain the following theorem.

Theorem 1 For $n \geq 1, r \geq 1,1 \leq k \leq n$, we have

$$
\left(\begin{array}{c}
r n+n-k-1 \\
n-k
\end{array}\right)(n-1)_{n-k}=(-1)^{n} \sum_{j=k}^{n} \sum_{l_{1}+\cdots+l_{n}=j-k} S_{2}(n, j)(-1)^{j} H_{l_{1}+1}^{(r)} \cdots H_{l_{n}+1}^{(r)}(j-1)_{j-k}
$$

We recall the following equation:

$$
\left(\frac{\log (1+t)}{t}\right)^{n}=\sum_{l=0}^{\infty} \frac{n !}{(l+n) !} S_{1}(l+n, n) t^{l}
$$

For $n \geq 1$, from (11), (17) and (18), we have

$$
\begin{aligned}
q_{n}(x) & =x\left(\frac{-\log (1-t)}{t(1-t)^{r}}\right)^{n} x^{-1}(-1)^{n} \phi_{n}(-x) \\
& =x\left(\frac{\log (1-t)}{-t}\right)^{n}(1-t)^{-r n} x^{-1}(-1)^{n} \phi_{n}(-x) \\
& =(-1)^{n} \sum_{j=1}^{n} S_{2}(n, j)(-1)^{j} x\left(\frac{\log (1-t)}{-t}\right)^{n}(1-t)^{-r n} x^{j-1} \\
& =(-1)^{n} \sum_{j=1}^{n} S_{2}(n, j)(-1)^{j} x\left(\frac{\log (1-t)}{-t}\right)^{n} \sum_{l=0}^{j-1}\left(\begin{array}{c}
r n+l-1 \\
l
\end{array}\right)(j-1)_{l} x^{j-1-l}
\end{aligned}
$$




$$
\begin{aligned}
= & (-1)^{n} \sum_{j=1}^{n} S_{2}(n, j)(-1)^{j} \sum_{l=0}^{j-1}\left(\begin{array}{c}
r n+l-1 \\
l
\end{array}\right)(j-1)_{l} x \sum_{m=0}^{j-1-l} \frac{n !}{(m+n) !} \\
& \times S_{1}(m+n, n)(-t)^{m} x^{j-1-l} \\
= & (-1)^{n} \sum_{j=1}^{n} \sum_{l=0}^{j-1} \sum_{m=0}^{j-1-l}(-1)^{j+m}\left(\begin{array}{c}
r n+l-1 \\
l
\end{array}\right) \frac{n !}{(m+n) !} \frac{(j-1) !}{(j-1-l-m) !} \\
& \times S_{1}(m+n, n) S_{2}(n, j) x^{j-l-m} \\
= & (-1)^{n} \sum_{k=1}^{n}\left\{\sum_{j=k}^{n} \sum_{l=0}^{j-k}(-1)^{k+l}\left(\begin{array}{c}
r n+l-1 \\
l
\end{array}\right) \frac{n !}{(j-l-k+n) !} \frac{(j-1) !}{(k-1) !}\right. \\
& \left.\times S_{1}(j-l-k+n, n) S_{2}(n, j)\right\} x^{k} .
\end{aligned}
$$

Therefore, by (19) and (23), we obtain the following theorem.

Theorem 2 For $r, n \geq 1,1 \leq k \leq n$, we have

$$
\begin{aligned}
& \left(\begin{array}{c}
r n+n-k-1 \\
n-k
\end{array}\right)(n-1)_{n-k} \\
& =(-1)^{n} \sum_{j=k}^{n} \sum_{l=0}^{j-k}(-1)^{k+l}\left(\begin{array}{c}
r n+l-1 \\
l
\end{array}\right) \frac{n !}{(j-l-k+n) !} \frac{(j-1) !}{(k-1) !} \\
& \quad \times S_{1}(j-l-k+n, n) S_{2}(n, j) .
\end{aligned}
$$

Here we invoke the following identity:

$$
\sum_{n=1}^{\infty}\left(\sum_{m=1}^{n} m H_{m}^{(r)}\right) t^{n}=\frac{t(1-r \log (1-t))}{(1-t)^{r+2}}
$$

Let us consider the following associated sequence:

$$
q_{n}(x) \sim\left(1, t(1-t)^{r+2}\right) .
$$

For $n \geq 1$, by (19) and (25), we get

$$
q_{n}(x)=\sum_{k=1}^{n}\left(\begin{array}{c}
(r+3) n-k-1 \\
n-k
\end{array}\right)(n-1)_{n-k} x^{k}
$$

Let us assume that

$$
p_{n}(x) \sim(1, t(1-r \log (1-t)))
$$

For $n \geq 1$, by (11), (27) and $x^{n} \sim(1, t)$, we get

$$
\begin{aligned}
p_{n}(x) & =7 x\left(\frac{t}{t(1-r \log (1-t))}\right)^{n} x^{-1} x^{n} \\
& =x(1-r \log (1-t))^{-n} x^{n-1}
\end{aligned}
$$




$$
\begin{aligned}
& =x \sum_{l=0}^{\infty}\left(\begin{array}{c}
n+l-1 \\
l
\end{array}\right) r^{l}(\log (1-t))^{l} x^{n-1} \\
& =x \sum_{l=0}^{n-1}\left(\begin{array}{c}
n+l-1 \\
l
\end{array}\right) r^{l} \sum_{j=0}^{n-1-l} \frac{l !}{(j+l) !} S_{1}(j+l, l) t^{j+l} x^{n-1} \\
& =\sum_{l=0}^{n-1} \sum_{j=0}^{n-1-l} l ! r^{l}\left(\begin{array}{c}
n+l-1 \\
l
\end{array}\right)\left(\begin{array}{c}
n-1 \\
j+l
\end{array}\right) S_{1}(j+l, l) x^{n-j-l} \\
& =\sum_{k=1}^{n}\left\{\sum_{l=0}^{n-k} l ! r^{l}\left(\begin{array}{c}
n+l-1 \\
l
\end{array}\right)\left(\begin{array}{c}
n-1 \\
k-1
\end{array}\right) S_{1}(n-k, l)\right\} x^{k} .
\end{aligned}
$$

For $n \geq 1$, from (11), (25) and (27), we can derive the following equation:

$$
\begin{aligned}
& q_{n}(x)=x\left(\frac{t(1-r \log (1-t))}{t(1-t)^{r+2}}\right)^{n} x^{-1} p_{n}(x) \\
& =x\left(\sum_{j=1}^{\infty}\left(\sum_{m=1}^{j} m H_{m}^{(r)}\right) t^{j-1}\right)^{n} \sum_{a=1}^{n}\left\{\sum_{l=0}^{n-a} l ! r^{l}\left(\begin{array}{c}
n+l-1 \\
l
\end{array}\right)\left(\begin{array}{c}
n-1 \\
a-1
\end{array}\right)\right. \\
& \left.\times S_{1}(n-a, l)\right\} x^{a-1} \\
& =\sum_{a=1}^{n}\left\{\sum_{l=0}^{n-a} l ! r^{l}\left(\begin{array}{c}
n+l-1 \\
l
\end{array}\right)\left(\begin{array}{c}
n-1 \\
a-1
\end{array}\right) S_{1}(n-a, l)\right\} \\
& \times x\left[\sum_{j=0}^{\infty}\left\{\sum_{j_{1}+\cdots+j_{n}=j}\left(\sum_{m_{1}=1}^{j_{1}+1} \cdots \sum_{m_{n}=1}^{j_{n}+1} m_{1} \cdots m_{n} H_{m_{1}}^{(r)} \cdots H_{m_{n}}^{(r)}\right)\right\} t^{j}\right] x^{a-1} \\
& =\sum_{a=1}^{n} \sum_{l=0}^{n-a} \sum_{k=1}^{a} \sum_{j_{1}+\cdots+j_{n}=a-k}\left(\sum_{m_{1}=1}^{j_{1}+1} \cdots \sum_{m_{n}=1}^{j_{n}+1} m_{1} \cdots m_{n} H_{m_{1}}^{(r)} \cdots H_{m_{n}}^{(r)}\right) \\
& \times l ! r^{l}\left(\begin{array}{c}
n+l-1 \\
l
\end{array}\right)\left(\begin{array}{c}
n-1 \\
a-1
\end{array}\right) S_{1}(n-a, l)(a-1)_{a-k} x^{k} \\
& =\sum_{k=1}^{n}\left\{\sum_{a=k}^{n} \sum_{l=0}^{n-a} \sum_{j_{1}+\cdots+j_{n}=a-k}\left(\sum_{m_{1}=1}^{j_{1}+1} \cdots \sum_{m_{n}=1}^{j_{n}+1} m_{1} \cdots m_{n} H_{m_{1}}^{(r)} \cdots H_{m_{n}}^{(r)}\right)\right. \\
& \left.\times l ! r^{l}\left(\begin{array}{c}
n+l-1 \\
l
\end{array}\right)\left(\begin{array}{c}
n-1 \\
a-1
\end{array}\right) S_{1}(n-a, l)(a-1)_{a-k}\right\} x^{k} .
\end{aligned}
$$

Therefore, by (26) and (29), we obtain the following theorem.

Theorem 3 For $n, r \geq 1,1 \leq k \leq n$, we have

$$
\begin{aligned}
& \left(\begin{array}{c}
(r+3) n-k-1 \\
n-k
\end{array}\right)(n-1)_{n-k} \\
& =\sum_{a=k}^{n} \sum_{l=0}^{n-a} \sum_{j_{1}+\cdots+j_{n}=a-k}\left(\sum_{m_{1}=1}^{j_{1}+1} \cdots \sum_{m_{n}=1}^{j_{n}+1} m_{1} \cdots m_{n} H_{m_{1}}^{(r)} \cdots H_{m_{n}}^{(r)}\right) l ! r^{l} \\
& \quad \times\left(\begin{array}{c}
n+l-1 \\
l
\end{array}\right)\left(\begin{array}{c}
n-1 \\
a-1
\end{array}\right) S_{1}(n-a, l)(a-1)_{n-k} .
\end{aligned}
$$


Here we use the following identity:

$$
\sum_{n=1}^{\infty} n H_{n}^{(r)} t^{n}=\frac{t(1-r \log (1-t))}{(1-t)^{r+1}}
$$

Let us consider the following associated sequence:

$$
q_{n}(x) \sim\left(1, t(1-t)^{r+1}\right) .
$$

For $n \geq 1$, from (19) and (31), we have

$$
q_{n}(x)=\sum_{k=1}^{n}\left(\begin{array}{c}
(r+2) n-k-1 \\
n-k
\end{array}\right)(n-1)_{n-k} x^{k}
$$

Let us assume that

$$
p_{n}(x) \sim(1, t(1-r \log (1-t))) \text {. }
$$

Then, from (28) and (33), we note that, for $n \geq 1$,

$$
p_{n}(x)=\sum_{k=1}^{n}\left\{\sum_{l=0}^{n-k} l ! r^{l}\left(\begin{array}{c}
n+l-1 \\
l
\end{array}\right)\left(\begin{array}{c}
n-1 \\
k-1
\end{array}\right) S_{1}(n-k, l)\right\} x^{k}
$$

For $n \geq 1$, by (11), (32) and (33), we get

$$
\begin{aligned}
& q_{n}(x)=x\left(\frac{t(1-r \log (1-t))}{t(1-t)^{r+1}}\right)^{n} x^{-1} p_{n}(x) \\
& =x\left(\sum_{j=1}^{\infty} j H_{j}^{(r)} t^{j-1}\right)^{n} x^{-1} \sum_{a=1}^{n}\left\{\sum_{l=0}^{n-a} l ! r^{l}\left(\begin{array}{c}
n+l-1 \\
l
\end{array}\right)\left(\begin{array}{c}
n-1 \\
a-1
\end{array}\right) S_{1}(n-a, l)\right\} x^{a} \\
& =\sum_{a=1}^{n}\left\{\sum_{l=0}^{n-a} l ! r^{l}\left(\begin{array}{c}
n+l-1 \\
l
\end{array}\right)\left(\begin{array}{c}
n-1 \\
a-1
\end{array}\right) S_{1}(n-a, l)\right\} \\
& \times x \sum_{j=0}^{a-1}\left(\sum_{j_{1}+\cdots+j_{n}=j}\left(j_{1}+1\right) \cdots\left(j_{n}+1\right) H_{j_{1}+1}^{(r)} \cdots H_{j_{n}+1}^{(r)}\right) t^{j} x^{a-1} \\
& =\sum_{a=1}^{n} \sum_{l=0}^{n-a} \sum_{j=0}^{a-1}\left(\sum_{j_{1}+\cdots+j_{n}=j}\left(j_{1}+1\right) \cdots\left(j_{n}+1\right) H_{j_{1}+1}^{(r)} \cdots H_{j_{n}+1}^{(r)}\right) l ! r^{l} \\
& \times\left(\begin{array}{c}
n+l-1 \\
l
\end{array}\right)\left(\begin{array}{c}
n-1 \\
a-1
\end{array}\right) S_{1}(n-a, l)(a-1)_{j} x^{a-j} \\
& =\sum_{k=1}^{n}\left\{\sum_{a=k}^{n} \sum_{l=0}^{n-a}\left(\sum_{j_{1}+\cdots+j_{n}=a-k}\left(j_{1}+1\right) \cdots\left(j_{n}+1\right) H_{j_{1}+1}^{(r)} \cdots H_{j_{n}+1}^{(r)}\right) l ! r^{l}\right. \\
& \left.\times\left(\begin{array}{c}
n+l-1 \\
l
\end{array}\right)\left(\begin{array}{c}
n-1 \\
a-1
\end{array}\right) S_{1}(n-a, l)(a-1)_{a-k}\right\} x^{k} .
\end{aligned}
$$

Therefore, by (32) and (35), we obtain the following theorem. 
Theorem 4 For $n, r \geq 1,1 \leq k \leq n$, we have

$$
\begin{aligned}
& \left(\begin{array}{c}
(r+2) n-k-1 \\
n-k
\end{array}\right)(n-1)_{n-k} \\
& =\sum_{a=k}^{n} \sum_{l=0}^{n-a}\left(\begin{array}{l}
\sum_{j_{1}+\cdots+j_{n}=a-k}\left(j_{1}+1\right) \cdots\left(j_{n}+1\right) H_{j_{1}+1}^{(r)} \cdots H_{j_{n}+1}^{(r)}
\end{array}\right) l ! r^{l} \\
& \quad \times\left(\begin{array}{c}
n+l-1 \\
l
\end{array}\right)\left(\begin{array}{c}
n-1 \\
a-1
\end{array}\right) S_{1}(n-a, l)(a-1)_{a-k} .
\end{aligned}
$$

Now, we utilize the following identity:

$$
\sum_{n=1}^{\infty}(n+1) H_{n} t^{n}=\frac{t-\log (1-t)}{(1-t)^{2}} .
$$

Let us consider the following associated sequence:

$$
q_{n}(x) \sim\left(1, t(1-t)^{2}\right) .
$$

For $n \geq 1$, from (19) and (37), we have

$$
q_{n}(x)=\sum_{k=1}^{n}\left(\begin{array}{c}
3 n-k-1 \\
n-k
\end{array}\right)(n-1)_{n-k} x^{k} .
$$

Let us assume that

$$
p_{n}(x) \sim(1, t-\log (1-t)) .
$$

We observe that

$$
t-\log (1-t)=t+\sum_{n=1}^{\infty} \frac{t^{n}}{n}=2 t+\sum_{n=2}^{\infty} \frac{t^{n}}{n} .
$$

From (11), (39), (40) and $x^{n} \sim(1, t)$, we can derive the following equation:

$$
\begin{aligned}
p_{n}(x)= & x\left(\frac{t}{2\left(t+\sum_{n=2}^{\infty} \frac{t^{n}}{n}\right)}\right)^{n} x^{-1} x^{n} \\
= & 2^{-n} x\left(1+\sum_{n=2}^{\infty} \frac{t^{n-1}}{2 n}\right)^{-n} x^{n-1} \\
= & 2^{-n} x \sum_{l=0}^{\infty}\left(\begin{array}{c}
-n \\
l
\end{array}\right)\left(\sum_{n=2}^{\infty} \frac{t^{n-1}}{2 n}\right)^{l} x^{n-1} \\
= & 2^{-n} x \sum_{l=0}^{n-1}(-1)^{l}\left(\begin{array}{c}
n+l-1 \\
l
\end{array}\right. \\
& \times \sum_{m=0}^{n-1-l} \sum_{m_{1}+\cdots+m_{l}=m} \frac{1}{2^{l}\left(m_{1}+2\right) \cdots\left(m_{l}+2\right)} t^{m+l} x^{n-1}
\end{aligned}
$$




$$
\begin{aligned}
& =2^{-n} \sum_{l=0}^{n-1} \sum_{m=0}^{n-1-l} \sum_{m_{1}+\cdots+m_{l}=m}\left(-\frac{1}{2}\right)^{l}\left(\begin{array}{c}
n+l-1 \\
l
\end{array}\right) \frac{(n-1)_{m+l}}{\left(m_{1}+2\right) \cdots\left(m_{l}+2\right)} x^{n-l-m} \\
& =2^{-n} \sum_{k=1}^{n}\left\{\sum_{l=0}^{n-k} \sum_{m_{1}+\cdots+m_{l}=n-l-k}\left(-\frac{1}{2}\right)^{l}\left(\begin{array}{c}
n+l-1 \\
l
\end{array}\right) \frac{(n-1)_{n-k}}{\left(m_{1}+2\right) \cdots\left(m_{l}+2\right)}\right\} x^{k} .
\end{aligned}
$$

For $n \geq 1$, by (11), (37), (39) and (41), we get

$$
\begin{aligned}
& q_{n}(x)=x\left(\frac{t-\log (1-t)}{t-(1-t)^{2}}\right)^{n} x^{-1} p_{n}(x) \\
& =x\left(\sum_{j=0}^{\infty}(j+2) H_{j+1} t^{j}\right)^{n} x^{-1} 2^{-n} \sum_{a=1}^{n}\left\{\sum_{l=0}^{n-a} \sum_{m_{1}+\cdots+m_{l}=n-l-a}\left(-\frac{1}{2}\right)^{l}\right. \\
& \left.\times\left(\begin{array}{c}
n+l-1 \\
l
\end{array}\right) \frac{(n-1)_{n-a}}{\left(m_{1}+2\right) \cdots\left(m_{l}+2\right)}\right\} x^{a} \\
& =2^{-n} \sum_{a=1}^{n}\left\{\sum_{l=0}^{n-a} \sum_{m_{1}+\cdots+m_{l}=n-l-a}\left(-\frac{1}{2}\right)^{l}\left(\begin{array}{c}
n+l-1 \\
l
\end{array}\right)\right. \\
& \left.\times \frac{(n-1)_{n-a}}{\left(m_{1}+2\right) \cdots\left(m_{l}+2\right)}\right\} x \sum_{j=0}^{a-1}\left(\sum_{j_{1}+\cdots+j_{n}=j}\left(j_{1}+2\right) \cdots\left(j_{n}+2\right)\right. \\
& \left.\times H_{j_{1}+1} \cdots H_{j_{n}+1}\right)(a-1)_{j} x^{a-1-j} \\
& =2^{-n} \sum_{a=1}^{n} \sum_{l=0}^{n-a} \sum_{k=1}^{a} \sum_{m_{1}+\cdots+m_{l}=n-l-a} \sum_{j_{1}+\cdots+j_{n}=a-k}\left(-\frac{1}{2}\right)^{l}\left(\begin{array}{c}
n+l-1 \\
l
\end{array}\right) \\
& \times \frac{(n-1)_{n-a}(a-1)_{a-k}}{\left(m_{1}+2\right) \cdots\left(m_{l}+2\right)}\left(j_{1}+2\right) \cdots\left(j_{n}+2\right) H_{j_{1}+1} \cdots H_{j_{n}+1} x^{k} \\
& =2^{-n} \sum_{k=1}^{n}\left\{\sum_{a=k}^{n} \sum_{l=0}^{n-a} \sum_{m_{1}+\cdots+m_{l}=n-l-a} \sum_{j_{1}+\cdots+j_{n}=a-k}\left(-\frac{1}{2}\right)^{l}\left(\begin{array}{c}
n+l-1 \\
l
\end{array}\right)\right. \\
& \left.\times \frac{(n-1)_{n-a}(a-1)_{a-k}}{\left(m_{1}+2\right) \cdots\left(m_{l}+2\right)}\left(j_{1}+2\right) \cdots\left(j_{n}+2\right) H_{j_{1}+1} \cdots H_{j_{n}+1}\right\} x^{k} .
\end{aligned}
$$

Therefore, by (38) and (42), we obtain the following theorem.

Theorem 5 For $n \geq 1,1 \leq k \leq n$, we have

$$
\begin{aligned}
& \left(\begin{array}{c}
3 n-k-1 \\
n-k
\end{array}\right)(n-1)_{n-k} \\
& =2^{-n} \sum_{a=k}^{n} \sum_{l=0}^{n-a} \sum_{m_{1}+\cdots+m_{l}=n-l-a} \sum_{j_{1}+\cdots+j_{n}=a-k}\left(-\frac{1}{2}\right)^{l}\left(\begin{array}{c}
n+l-1 \\
l
\end{array}\right) \\
& \quad \times \frac{(n-1)_{n-a}(a-1)_{a-k}}{\left(m_{1}+2\right) \cdots\left(m_{l}+2\right)}\left(j_{1}+2\right) \cdots\left(j_{n}+2\right) H_{j_{1}+1} \cdots H_{j_{n+1}} .
\end{aligned}
$$


Now, we recall the following identity:

$$
\sum_{n=1}^{\infty} n^{2} H_{n} t^{n}=\frac{t\{1+2 t-(1+t) \log (1-t)\}}{(1-t)^{3}}
$$

Let us consider the following associated sequence:

$$
q_{n}(x) \sim\left(1, t(1-t)^{3}\right)
$$

For $n \geq 1$, from (19) and (44), we can derive the following equation:

$$
q_{n}(x)=\sum_{k=1}^{n}\left(\begin{array}{c}
4 n-k-1 \\
n-k
\end{array}\right)(n-1)_{n-k} x^{k} .
$$

Let us assume that

$$
p_{n}(x) \sim(1, t\{1+2 t-(1+t) \log (1-t)\}) .
$$

We observe that

$$
\begin{aligned}
1+2 t-(1+t) \log (1-t) & =1+2 t+(1+t) \sum_{j=1}^{\infty} \frac{t^{j}}{j} \\
& =1+2 t+t+\sum_{j=2}^{\infty} \frac{t^{j}}{j}+\sum_{j=1}^{\infty} \frac{t^{j+1}}{j} \\
& =1+3 t+\sum_{j=0}^{\infty} \frac{t^{j+2}}{j+2}+\sum_{j=0}^{\infty} \frac{t^{j+2}}{j+1} \\
& =1+3 t+\sum_{j=0}^{\infty} \frac{2 j+3}{(j+2)(j+1)} t^{j+2} .
\end{aligned}
$$

For $n \geq 1$, by (11), (46), (47) and $x^{n} \sim(1, t)$, we get

$$
\begin{aligned}
p_{n}(x)= & x\left(\frac{t}{t\{1+2 t-(1+t) \log (1-t)\}}\right)^{n} x^{-1} x^{n} \\
= & x\left(1+3 t+\sum_{j=0}^{\infty} \frac{2 j+3}{(j+1)(j+2)} t^{j+2}\right)^{-n} x^{n-1} \\
= & x \sum_{l=0}^{n-1}(-1)^{l}\left(\begin{array}{c}
n+l-1 \\
l
\end{array}\right)\left(3+\sum_{j=0}^{\infty} \frac{2 j+3}{(j+1)(j+2)} t^{j+1}\right)^{l} t^{l} x^{n-1} \\
= & \sum_{l=0}^{n-1} \sum_{a=0}^{n-1-l n-a-l} \sum_{k=1}^{n j_{1}+\cdots+j_{a}=n-a-k-l}(-1)^{l}\left(\begin{array}{c}
n+l-1 \\
l
\end{array}\right)\left(\begin{array}{l}
l \\
a
\end{array}\right) 3^{l-a}(n-1)_{n-k} \\
& \times\left(\frac{\prod_{i=1}^{a}\left(2 j_{i}+3\right)}{\prod_{i=1}^{a}\left(j_{i}+1\right)\left(j_{i}+2\right)}\right) x^{k}
\end{aligned}
$$


For $n \geq 1$, from (11), (44), (46) and (48), we have

$$
\begin{aligned}
q_{n}(x)= & x\left(\frac{t(1+2 t-(1+t) \log (1-t))}{t(1-t)^{3}}\right)^{n} x^{-1} p_{n}(x) \\
= & \sum_{m=1}^{n} \sum_{l=0}^{n-m} \sum_{a=0}^{n-m-l} \sum_{j_{1}+\cdots+j_{a}=n-a-m-l}(-1)^{l}\left(\begin{array}{c}
n+l-1 \\
l
\end{array}\right)\left(\begin{array}{l}
l \\
a
\end{array}\right) 3^{l-a}(n-1)_{n-m} \\
& \times\left(\frac{\prod_{i=1}^{a}\left(2 j_{i}+3\right)}{\prod_{i=1}^{a}\left(j_{i}+1\right)\left(j_{i}+2\right)}\right) x \sum_{b=0}^{m-1} \sum_{b_{1}+\cdots+b_{n}=b}\left(\prod_{i=1}^{n}\left(b_{i}+1\right)^{2} H_{b_{i}+1}\right) t^{b} x^{m-1} \\
= & \sum_{m=1}^{n} \sum_{l=0}^{n-m} \sum_{a=0}^{n-m-l} \sum_{j_{1}+\cdots+j_{a}=n-a-m-l}(-1)^{l}\left(\begin{array}{c}
n+l-1 \\
l
\end{array}\right)\left(\begin{array}{l}
l \\
a
\end{array}\right) 3^{l-a}(n-1)_{n-m} \\
& \times\left(\frac{\prod_{i=1}^{a}\left(2 j_{i}+3\right)}{\prod_{i=1}^{a}\left(j_{i}+1\right)\left(j_{i}+2\right)}\right) \sum_{b=0}^{m-1} \sum_{b_{1}+\cdots+b_{n}=b} \prod_{i=1}^{n}\left(b_{i}+1\right)^{2} H_{b_{i}+1}(m-1)_{b} x^{m-b} \\
= & \sum_{k=1}^{n}\left\{\sum_{m=k}^{n} \sum_{l=0}^{n-m} \sum_{a=0}^{n-m-l} \sum_{j_{1}+\cdots+j_{a}=n-a-m-l} \sum_{b_{1}+\cdots+b_{n}=m-k}(-1)^{l}\left(\begin{array}{c}
n+l-1 \\
l
\end{array}\right)\left(\begin{array}{l}
l \\
a
\end{array}\right)\right. \\
& \left.\times 3^{l-a}(n-1)_{n-m}(m-1)_{m-k}\left(\frac{\prod_{i=1}^{a}\left(2 j_{i}+3\right) \prod_{i=1}^{n}\left(b_{i}+1\right)^{2} H_{b_{i}+1}}{\prod_{i=1}^{a}\left(j_{i}+1\right)\left(j_{i}+2\right)}\right)\right\} x^{k} .
\end{aligned}
$$

Therefore, by (45) and (49), we obtain the following theorem.

Theorem 6 For $n \geq 1,1 \leq k \leq n$, we have

$$
\begin{aligned}
& \left(\begin{array}{c}
4 n-k-1 \\
n-k
\end{array}\right)(n-1)_{n-k} \\
& =\sum_{m=k}^{n} \sum_{l=0}^{n-m} \sum_{a=0}^{n-m-l} \sum_{j_{1}+\cdots+j_{a}=n-a-m-l b_{1}+\cdots+b_{n}=m-k}(-1)^{l}\left(\begin{array}{c}
n+l-1 \\
l
\end{array}\right)\left(\begin{array}{l}
l \\
a
\end{array}\right) 3^{l-a} \\
& \quad \times(n-1)_{n-m}(m-1)_{m-k}\left(\frac{\prod_{i=1}^{a}\left(2 j_{i}+3\right) \prod_{i=1}^{n}\left(b_{i}+1\right)^{2} H_{b_{i}+1}}{\prod_{i=1}^{a}\left(j_{i}+1\right)\left(j_{i}+2\right)}\right)
\end{aligned}
$$

Here we invoke the following identity:

$$
\sum_{b=1}^{\infty}\left(\sum_{c=1}^{b} c^{2} H_{c}\right) t^{b}=\frac{t\{1+2 t-(1+t) \log (1-t)\}}{(1-t)^{4}}
$$

Let us consider the following associated sequence:

$$
q_{n}(x) \sim\left(1, t(1-t)^{4}\right) .
$$


From (19) and (51), we note that

$$
q_{n}(x)=\sum_{k=1}^{n}\left(\begin{array}{c}
5 n-k-1 \\
n-k
\end{array}\right)(n-1)_{n-k} x^{k} .
$$

Let us assume that

$$
p_{n}(x) \sim(1, t(1+2 t-(1+t) \log (1-t))) .
$$

For $n \geq 1$, from (48) and (49), we have

$$
\begin{aligned}
p_{n}(x)= & \sum_{k=1}^{n}\left\{\sum_{l=0}^{n-k} \sum_{a=0}^{n-k-l} \sum_{j_{1}+\cdots+j_{a}=n-a-k-l}(-1)^{l}\left(\begin{array}{c}
n+l-1 \\
l
\end{array}\right)\left(\begin{array}{l}
l \\
a
\end{array}\right) 3^{l-a}(n-1)_{n-k}\right. \\
& \left.\times\left(\frac{\prod_{i=1}^{a}\left(2 j_{i}+3\right)}{\prod_{i=1}^{a}\left(j_{i}+1\right)\left(j_{i}+2\right)}\right)\right\} x^{k} .
\end{aligned}
$$

For $n \geq 1$, from (11), (51), (53) and (50), we can derive the following identity:

$$
\begin{aligned}
& q_{n}(x)=x\left(\frac{t\{1+2 t-(1+t) \log (1-t)\}}{t(1-t)^{4}}\right)^{n} x^{-1} p_{n}(x) \\
& =x\left(\sum_{b=0}^{\infty}\left(\sum_{c=1}^{b+1} c^{2} H_{c}\right) t^{b}\right)^{n} x^{-1} p_{n}(x) \\
& =x \sum_{b=0}^{\infty} \sum_{b_{1}+\cdots+b_{n}=b}\left\{\sum_{c_{1}=1}^{b_{1}+1} \cdots \sum_{c_{n}=1}^{b_{n}+1} c_{1}^{2} \cdots c_{n}^{2} H_{c_{1}} \cdots H_{c_{n}}\right\} t^{b} \\
& \times \sum_{m=1}^{n}\left\{\sum_{l=0}^{n-m} \sum_{a=0}^{n-m-l} \sum_{j_{1}+\cdots+j_{a}=n-a-m-l}(-1)^{l}\left(\begin{array}{c}
n+l-1 \\
l
\end{array}\right)\left(\begin{array}{l}
l \\
a
\end{array}\right) 3^{l-a}\right. \\
& \left.\times(n-1)_{n-m}\left(\frac{\prod_{i=1}^{a}\left(2 j_{i}+3\right)}{\prod_{i=1}^{a}\left(j_{i}+1\right)\left(j_{i}+2\right)}\right)\right\} x^{m-1} \\
& =\sum_{m=1}^{n} \sum_{l=0}^{n-m} \sum_{a=0}^{n-m-l} \sum_{j_{1}+\cdots+j_{a}=n-a-m-l}(-1)^{l}\left(\begin{array}{c}
n+l-1 \\
l
\end{array}\right)\left(\begin{array}{l}
l \\
a
\end{array}\right) 3^{l-a}(n-1)_{n-m} \\
& \times\left(\frac{\prod_{i=1}^{a}\left(2 j_{i}+3\right)}{\prod_{i=1}^{a}\left(j_{i}+1\right)\left(j_{i}+2\right)}\right) \sum_{b=0}^{m-1} \sum_{b_{1}+\cdots+b_{n}=b}\left\{\sum_{c_{1}=1}^{b_{1}+1} \cdots \sum_{c_{n}=1}^{b_{n}+1} c_{1}^{2} \cdots c_{n}^{2} H_{c_{1}} \cdots H_{c_{n}}\right\} \\
& \times(m-1)_{b} x^{m-b} \\
& =\sum_{k=1}^{n}\left\{\sum_{m=k}^{n} \sum_{l=0}^{n-m} \sum_{a=0}^{n-m-l} \sum_{j_{1}+\cdots+j_{a}=n-a-m-l} \sum_{b_{1}+\cdots+b_{n}=m-k}(-1)^{l}\left(\begin{array}{c}
n+l-1 \\
l
\end{array}\right)\right. \\
& \times\left(\begin{array}{l}
l \\
a
\end{array}\right) 3^{l-a}(n-1)_{n-m}(m-1)_{m-k}\left(\frac{\prod_{i=1}^{a}\left(2 j_{i}+3\right)}{\prod_{i=1}^{a}\left(j_{i}+1\right)\left(j_{i}+2\right)}\right) \\
& \left.\times \sum_{c_{1}=1}^{b_{1}+1} \cdots \sum_{c_{n}=1}^{b_{n}+1} \prod_{i=1}^{n} c_{i}^{2} H_{c_{i}}\right\} x^{k}
\end{aligned}
$$

Therefore, by (52) and (55), we obtain the following theorem. 
Theorem 7 For $n \geq 1,1 \leq k \leq n$, we have

$$
\begin{aligned}
& \left(\begin{array}{c}
5 n-k-1 \\
n-k
\end{array}\right)(n-1)_{n-k} \\
& =\sum_{m=k}^{n} \sum_{l=0}^{n-m} \sum_{a=0}^{n-m-l} \sum_{j_{1}+\cdots+j_{a}=n-a-m-l} \sum_{b_{1}+\cdots+b_{n}=m-k}(-1)^{l}\left(\begin{array}{c}
n+l-1 \\
l
\end{array}\right)\left(\begin{array}{l}
l \\
a
\end{array}\right) 3^{l-a} \\
& \quad \times(n-1)_{n-m}(m-1)_{m-k}\left(\frac{\prod_{i=1}^{a}\left(2 j_{i}+3\right)}{\prod_{i=1}^{a}\left(j_{i}+1\right)\left(j_{i}+2\right)}\right) \sum_{c_{1}=1}^{b_{1}+1} \cdots \sum_{c_{n}=1}^{b_{n}+1} \prod_{i=1}^{n} c_{i}^{2} H_{c_{i}} .
\end{aligned}
$$

Here we use the following identity:

$$
\sum_{n=1}^{\infty} n(2 n+1) H_{n} t^{n}=\frac{t\{3(1+t)-(t+3) \log (1-t)\}}{(1-t)^{3}} .
$$

Let us consider the following associated sequence:

$$
q_{n}(x) \sim\left(1, t(1-t)^{3}\right) .
$$

By (19) and (57), we get

$$
q_{n}(x)=\sum_{k=1}^{n}\left(\begin{array}{c}
4 n-k-1 \\
n-k
\end{array}\right)(n-1)_{n-k} x^{k} \quad(n \geq 1) .
$$

Let us assume that

$$
p_{n}(x) \sim(1, t\{3(1+t)-(t+3) \log (1-t)\}) .
$$

We see that

$$
3(1+t)-(t+3) \log (1-t)=3+6 t+\sum_{n=1}^{\infty} \frac{4 n+1}{n(n+1)} t^{n+1}
$$

For $n \geq 1$, from (11), (59), (60) and $x^{n} \sim(1, t)$, we have

$$
\begin{aligned}
p_{n}(x) & =x\left(\frac{t}{t\{3(1+t)-(t+3) \log (1-t)\}}\right)^{n} x^{-1} x^{n} \\
& =x(3(1+t)-(t+3) \log (1-t))^{-n} x^{n-1} \\
& =x\left(3+6 t+\sum_{j=1}^{\infty} \frac{4 j+1}{j(j+1)} t^{j+1}\right)^{-n} x^{n-1} .
\end{aligned}
$$

From (61), by the same method of (48), we get

$$
\begin{aligned}
p_{n}(x)= & 3^{-n} \sum_{k=1}^{n}\left\{\sum_{l=0}^{n-k} \sum_{a=0}^{n-k-l} \sum_{j_{1}+\cdots+j_{a}=n-a-l-k}(-1)^{l}\left(\begin{array}{c}
n+l-1 \\
l
\end{array}\right)\left(\begin{array}{l}
l \\
a
\end{array}\right) 2^{l-a}\right. \\
& \left.\times(n-1)_{n-k}\left(\prod_{i=1}^{a} \frac{\left(4 j_{i}+5\right)}{3\left(j_{i}+1\right)\left(j_{i}+2\right)}\right)\right\} x^{k} .
\end{aligned}
$$


For $n \geq 1$, by (11), (56), (57), (59) and (62), we get

$$
\begin{aligned}
q_{n}(x)= & x\left(\frac{t\{3(1+t)-(t+3) \log (1-t)\}}{t(1-t)^{3}}\right)^{n} x^{-1} p_{n}(x) \\
= & x\left(\sum_{b=0}^{\infty}(b+1)(2 b+3) H_{b+1} t^{b}\right)^{n} x^{-1} p_{n}(x) \\
= & x \sum_{b=0}^{\infty}\left(\sum_{b_{1}+\cdots+b_{n}=b}\left(\prod_{i=1}^{b}\left(b_{i}+1\right)\left(2 b_{i}+3\right) H_{b_{i}+1}\right) t^{b}\right) \\
& \times 3^{-n} \sum_{m=1}^{n}\left\{\sum_{l=0}^{n-m} \sum_{a=0}^{n-m-l} \sum_{j_{1}+\cdots+j_{a}=n-a-l-m}(-1)^{l}\left(\begin{array}{l}
n+l-1 \\
l
\end{array}\right)\left(\begin{array}{l}
l \\
a
\end{array}\right) 2^{l-a}\right. \\
& \left.\times(n-1)_{n-m} \prod_{i=1}^{a} \frac{\left(4 j_{i}+5\right)}{3\left(j_{i}+1\right)\left(j_{i}+2\right)}\right\}^{m-1}(-1)^{l}\left(\begin{array}{l}
n+l-1 \\
l
\end{array}\right)\left(\begin{array}{l}
l \\
a
\end{array}\right) 2^{l-a}(n-1)_{n-m} \\
= & 3^{-n} \sum_{m=1}^{n} \sum_{l=0}^{n-m} \sum_{a=0}^{n-m-l} \sum_{j_{1}+\cdots+j_{a}=n-a-l-m} \\
& \times\left(\prod _ { i = 1 } ^ { a } \frac { ( 4 j _ { i } + 5 ) } { 3 ( j _ { i } + 1 ) ( j _ { i } + 2 ) } \sum _ { b = 0 } ^ { m - 1 } \sum _ { b _ { 1 } + \cdots + b _ { n } = b } \left(\prod_{i=1}^{n}\left(b_{i}+1\right)\left(2 b_{i}+3\right) H_{b_{i}+1} x^{m-b} .\right.\right.
\end{aligned}
$$

By the same method, we can derive the following identity from (63):

$$
\begin{aligned}
& q_{n}(x)=3^{-n} \sum_{k=1}^{n}\left\{\sum_{m=k}^{n} \sum_{l=0}^{n-m} \sum_{a=0}^{n-m-l} \sum_{j_{1}+\cdots+j_{a}=n-a-l-m} \sum_{b_{1}+\cdots+b_{n}=m-k}(-1)^{l}\right. \\
& \times\left(\begin{array}{c}
n+l-1 \\
l
\end{array}\right)\left(\begin{array}{l}
l \\
a
\end{array}\right) 2^{l-a}(n-1)_{n-m}(m-1)_{m-k}\left(\prod_{i=1}^{a} \frac{\left(4 j_{i}+5\right)}{3\left(j_{i}+1\right)\left(j_{i}+2\right)}\right) \\
& \left.\times \prod_{i=1}^{n}\left(b_{i}+1\right)\left(2 b_{i}+3\right) H_{b_{i}+1}\right\} x^{k} \text {. }
\end{aligned}
$$

By comparing coefficients on both sides of (58) and (64), we get

$$
\begin{aligned}
& \left(\begin{array}{c}
4 n-k-1 \\
n-k
\end{array}\right)(n-1)_{n-k} \\
& =3^{-n} \sum_{m=k}^{n} \sum_{l=0}^{n-m} \sum_{a=0}^{n-m-l} \sum_{j_{1}+\cdots+j_{a}=n-a-l-m} \sum_{b_{1}+\cdots+b_{n}=m-k}(-1)^{l}\left(\begin{array}{c}
n+l-1 \\
l
\end{array}\right)\left(\begin{array}{l}
l \\
a
\end{array}\right) \\
& \quad \times 2^{l-a}(n-1)_{n-m}(m-1)_{m-k}\left(\prod_{i=1}^{a} \frac{\left(4 j_{i}+5\right)}{3\left(j_{i}+1\right)\left(j_{i}+2\right)}\right) \\
& \quad \times\left(\prod_{i=1}^{n}\left(b_{i}+1\right)\left(2 b_{i}+3\right) H_{b_{i}+1}\right) .
\end{aligned}
$$


Remark Recently, several authors have studied the $q$-extension of harmonic and hyperharmonic numbers (see [11-13]).

Competing interests

The authors declare that they have no competing interests.

Authors' contributions

All authors contributed equally to the manuscript and typed, read and approved the final manuscript.

\section{Author details}

${ }^{1}$ Department of Mathematics, Sogang University, Seoul, 121-742, Republic of Korea. ${ }^{2}$ Department of Mathematics, Kwangwoon University, Seoul, 139-701, Republic of Korea.

\section{Acknowledgements}

The authors express their sincere gratitude to the referees for their valuable suggestions and comments. This work was supported by the National Research Foundation of Korea (NRF) grant funded by the Korea government (MOE) (No. 2012R1A1A2003786)

Received: 26 April 2013 Accepted: 22 July 2013 Published: 7 August 2013

\section{References}

1. Roman, S: More on the umbral calculus, with emphasis on the $q$-umbral calculus. J. Math. Anal. Appl. 107, 222-254 (1985)

2. Roman, S: The Umbral Calculus. Dover, New York (2005)

3. Araci, S, Acikgoz, M, Kilicman, A: Extended $p$-adic $q$-invariant integrals on $\mathbb{Z}_{p}$ associated with applications of umbral calculus. Adv. Differ. Equ. 2013, 96 (2013)

4. Kim, DS, Kim, T, Lee, S-H, Rim, S-H: Some identities of Bernoulli, Euler and Abel polynomials arising from umbral calculus. Adv. Differ. Equ. 2013, 15 (2013)

5. Kim, DS, Kim, T, Dolgy, DV, Rim, S-H: Some new identities of Bernoulli, Euler and Hermite polynomials arising from umbral calculus. Adv. Differ. Equ. 2013, 73 (2013)

6. Kim, DS, Kim, T, Lee, S-H, Rim, S-H: A note on the higher-order Frobenius-Euler polynomials and Sheffer sequences. Adv. Differ. Equ. 2013, 41 (2013)

7. Dere, R, Simsek, Y: Applications of umbral algebra to some special polynomials. Adv. Stud. Contemp. Math. 22(3), 433-438 (2012)

8. Kim, DS, Kim, T: Some identities of Frobenius-Euler polynomials arising from umbral calculus. Adv. Differ. Equ. 2012, $196(2012)$

9. Dil, A, Kurt, V: Polynomials related to harmonic numbers and evaluation of harmonic number series. Integers 12,38 (2012)

10. Dil, A, Kurt, V: Polynomials related to harmonic numbers and evaluation of harmonic number series II. Appl. Anal. Discrete Math. 5, 212-229 (2011)

11. Mansour, T, Shattuck, M, Song, C: q-Analogs of identities involving harmonic numbers and binomial coefficients. Appl. Appl. Math. 7(1), 22-36 (2012)

12. Mansour, T: Identities on harmonic and q-harmonic number sums. Afr. Math. 23, 135-143 (2012)

13. Mansour, T, Shattuck, M: A q-analog of the hyperharmonic numbers. Afr. Math. (2012). doi:10.1007/s13370-012-0106-6

doi:10.1186/1687-1847-2013-235

Cite this article as: Kim and Kim: Identities involving harmonic and hyperharmonic numbers. Advances in Difference Equations 2013 2013:235.

\section{Submit your manuscript to a SpringerOpen ${ }^{\circ}$ journal and benefit from:}

- Convenient online submission

- Rigorous peer review

- Immediate publication on acceptance

- Open access: articles freely available online

- High visibility within the field

- Retaining the copyright to your article 